bilateral connections with various motor neurones in the metathorax (Fig. 1a) ensuring rapid extension of the tibia, with inhibition of the antagonistic flexor muscles and of other systems. The distribution of the axon in the metathorax as revealed in the paper under review (Fig. 1b) shows striking agreement with the conclusions reached by electrophysiological methods alone. There are no major branches to be seen in the metathoraxic ganglia which cannot be accounted for by the known connections with motorneurones involved in the jump. It seems likely that this is the most important function of the descending movement detector neurones. There are also restricted connections with neurones in the prothorax and mesothorax, whose role in the jump are at present unknown. There are considerable individual differences in the detailed arrangements of these neurones. Assuming, as the authors do, that these differences are genetically determined, they may well form the basis of a behaviour variance, in the population, subject to natural selection.

O'Shea et al. conclude by pointing out that by using the methods of iontophoresis of cobalt ions, neural anatomists could uncover rich new fields for physiological investigation.

\section{Mapping of poliovirus receptor}

\section{from our \\ Molecular Genetics Correspondent}

Poliovirus provides one of those interesting situations in which the susceptibility of a cell to a virus may depend upon its possession of the appropriate receptor. Human cells possess the receptor and can therefore be killed by the virus; whereas rodent cells usually cannot be infected. But in human-rodent hybrid cells, possession of a human gene for the poliovirus receptor is sufficient to enable the virus nucleic acid to enter the cell and, once this first step has been taken, the virus can then multiply without the mediation of any further human gene products, the rodent genetic apparatus being sufficient for its needs. It should therefore in principle be possible to identify the human chromosome(s) carrying the gene(s) concerned with poliovirus reception by making use of humanrodent hybrids which differ in their complement of human chromosomes; when some of the human chromosomes have been shed after hybridisation, loss of the appropriate human chromosome(s) should mean that the hybrids also lose their suspectibility to poliovirus. By performing such experiments, Miller et al. report in the April issue of Cell (1, 167; 1974) that a single human chromosome is responsible for conferring upon cells the ability to accept poliovirus.

These experiments have succeeded where earlier attempts to gain this information failed because of two recent developments; hybrid lines are now available with a high degree of homogeneity of human chromosomes (necessary to establish correlations with poliovirus sensitivity) and banding techniques can be used to identify these chromosomes from the others of the set. Virus-resistant and virus-sensitive human-mouse hybrid cells all proved to possess about the same number of human and mouse chromosomes, excluding the possibility that changes in the ratios of two genomes might be relevant in establishing susceptibility to the virus (a mechanism important in polyoma susceptibility or adenovirus multiplication).

The chromosomes of several poliovirus resistant and sensitive lines were examined by both fluorescent $\mathrm{Q}$ banding, which produces characteristic striations of each human and mouse chromosome, and also by $\mathrm{C}$ banding, which stains principally the centrometric heterochromatin. Cell lines resistant to poliovirus lacked human chromosome 19, whereas lines sensitive to infection possessed this chromosome. This implies that chromosome 19 carries all the information necessary for acceptance of poliovirus by a human cell. This does not prove, of course, that only a single gene codes for the receptor protein; but this is the most likely interpretation of these results since the genes carried on several chromosomes were responsible, the loss of each of these chromosomes might produce the receptor-deficient phenotype. The idea that more than one gene might be involved, but that all may be located on chromosome 19, may be excluded in future experiments.

\section{Interference in heavy ion inelastic scattering}

from our

Nuclear Theory Correspondent

ONE OF THE most interesting features of heavy ion interactions is the interference effects between the different processes that can take place when the interacting ions differ by only a few nucleons. For example, if ${ }^{16} \mathrm{O}$ is scattered by ${ }^{17} \mathrm{O}$, the neutron transfer reaction from ${ }^{17} \mathrm{O}$ to the ground state of ${ }^{16} \mathrm{O}$ gives the same emerging particles as the simple elastic scattering and so is indistinguishable from it. The corresponding amplitudes interfere quantum mechanically and if they are of comparable

\section{New MRC unit}

ThE Medical Research Council set up on April 1 a Mammalian Development Unit in University College, London. Its head is $\mathrm{Dr}$ Anne McLaren who previously worked in the Agricultural Research Council Unit of Animal Genetics in Edinburgh. She will be assisted initially by three other senior scientists two of whom are Dr M. H. L. Snow, also from the ARC Unit of Animal Genetics, and Dr D. G. Whittingham who is at present attached to the Physiological Laboratory in Cambridge.

The unit will study the growth and differentiation of the mammalian embryo, concentrating on the expression of genes in the early post-implantation embryo. Some of this work will be done in collaboration with Professor H. Harris. Dr McLaren will continue to use mice as her main experimental animals.

Dr Whittingham plans to extend his work on the low temperature storage of mouse embryos in collaboration with Dr Mary Lyon. The practical application of this work is in livestock breeding but many experiments, such as those on the mutagenic effects of irradiation on the stored embryos, can only be done with an animal whose genetics are well understood.

The new unit is being housed in the former home of Professor $\mathrm{H}$. Grüneberg's Experimental Genetics Unit which was disbanded in 1972. Its budget is $£ 175,000$ for five years, unless it expands quickly enough to need more.

magnitude this is evident in the observed elastic scattering cross sections.

Interference phenomena are sensitive to the relative amplitudes and phases of the contributing process so they can provide a detailed check of the models used to calculate them, in this case of the distorted wave theory of the interaction and the optical potentials used, as well as of the spectroscopic factors used to obtain the transfer amplitude. Many studies of elastic scattering of similar ions are being made with these aims in mind.

A group at the Max Planck Institute for Nuclear Physics in Heidelberg (Gelbke, Baur, Bock, Braun-Munzinger, Grochulski, Harvey and Stock, Nucl. Phys., A219, 253; 1974) has recently obtained evidence for the occurrence of similar interference phenomena in the inelastic scattering of similar heavy ions. They bombarded ${ }^{16} \mathrm{O}$ with ${ }^{17} \mathrm{O}$ ions with energies ranging from 22 to $32 \mathrm{MeV}$ and measured the cross section for the 